\title{
Threshold functions for substructures in random subsets of finite vector spaces
}

\author{
Changhao Chen* \\ Department of Mathematics \\ The Chinese University of Hong Kong \\ Shatin, N.T., Hong Kong \\ changhao. chenm@gmail . com
}

\author{
Catherine Greenhill ${ }^{\dagger}$ \\ School of Mathematics and Statistics \\ UNSW Sydney \\ Sydney NSW 2052, Australia \\ c.greenhill@unsw.edu.au
}

\author{
1 April 2020 \\ Mathematics Subject Classifications: 05B25, 60C05
}

\begin{abstract}
The study of substructures in random objects has a long history, beginning with Erdős and Rényi's work on subgraphs of random graphs. We study the existence of certain substructures in random subsets of vector spaces over finite fields. First we provide a general framework which can be applied to establish coarse threshold results and prove a limiting Poisson distribution at the threshold scale. To illustrate our framework we apply our results to $k$-term arithmetic progressions, sums, right triangles, parallelograms and affine planes. We also find coarse thresholds for the property that a random subset of a finite vector space is sum-free, or is a Sidon set.
\end{abstract}

Keywords: Finite vector space, threshold property, Poisson distribution

\section{Introduction}

Thresholds for the existence of substructures in random objects have been well studied in many contexts, for example in random graphs (see $[1,5,18]$ ), random subsets of integers (see $[31,34]$ ) and even in random fractal sets [36] where the threshold functions are taken with respect to various dimensions of fractal geometry. While extremal problems ask for conditions which guarantee that a certain substructure is always present, the random version relaxes this to ask that the substructure is present with probability which tends to 1 .

\footnotetext{
*Supported by Australian Research Council Discovery Project 170100786.

${ }^{\dagger}$ Supported by Australian Research Council Discovery Project 140101519.
} 
Let $G(n, p)$ denote the binomial random graph with $n$ vertices. The threshold for the property that $G(n, p)$ contains a given graph $G$ as a subgraph depends on the density of the densest subgraph of $G$. Formally, define

$$
m(G)=\max \{\rho(H) \mid H \subseteq G\}
$$

where $\subseteq$ denotes the subgraph relation and $\rho(H)=|E(H)| /|H|$ is the density of $H$. If $m(G)=\rho(G)$ then $G$ is balanced, and if $\rho(H)<\rho(G)$ for all proper subgraphs $H$ of $G$ then $G$ is stricly balanced. As part of their foundational work on random graphs, Erdös and Rényi [15] found the threshold for almost-sure containment of $G$ as a subgraph in $G(n, p)$, when $G$ is balanced. This was extended to general graphs by Bollobás [4], who showed that if $m(G) \geq 1$ then

$$
\lim _{n \rightarrow \infty} \operatorname{Pr}(G \subseteq G(n, p))= \begin{cases}0 & \text { if } p n^{1 / m(G)} \rightarrow 0 \\ 1 & \text { if } p n^{1 / m(G)} \rightarrow \infty\end{cases}
$$

Attention then turned to the distribution of the number of copies of a given graph in $G(n, p)$. Independently, Bollobás [4] and Karoński and Ruciński [25] showed that if $G$ is strictly balanced then, at the threshold scale (that is, when $p=c n^{-1 / m(G)}$ for some constant $c>0)$, the number of copies of $G$ in $G(n, p)$ tends to a Poisson distribution. Ruciński and Vince proved and the converse is true [33]. If $G$ is balanced but not strictly balanced then the limiting distribution is normal, as proved by Ruciński [32]. Analogous results (on the threshold for existence, and limiting Poisson distrubution) for subgraphs of random regular graphs were proved by Kim, Sudakov and $\mathrm{Vu}$ [26].

Researchers in arithmetic combinatorics have studied the existence of substructures in random sets of integers. Several important structures, such as arithmetic progressions, sum-free sets and Sidon sets, can be described in terms of solutions (or non-solutions) of a certain system of linear equations. Rué, Spiegel and Zumalacárregui [34] gave a threshold function for the existence of solutions to a given system of linear equations in random subsets of $[n]=\{1,2, \ldots, n\}$. In their result, the integer matrix $M$ representing the system of linear equations must have full rank, at least one solution with all entries positive, and for each $i \neq j$ there must be a solution $\boldsymbol{x}=\left(x_{1}, \ldots, x_{m}\right)$ with $x_{i} \neq x_{j}$. The threshold depends on a parameter $c(M)$ which is reminiscent of the parameter $m(G)$ arising in the study of subgraphs in random graphs [15]. In particular, they show that almost all subsets of $[n]$ of size $\omega\left(n^{1-2 / k}\right)$ contain arithmetic progressions of length $k$. Rué et al. also established a Poisson limiting distribution for the number of solutions of the linear system $M \boldsymbol{x}=\mathbf{0}$, at the threshold scale.

A set $S \subseteq \mathbb{N}$ is a Sidon set if all sums of the form $x+y$ with $x \leq y$ and $x, y \in S$ are distinct. Kohayakawa, Lee, Rödl and Samotij [28] analysed the maximum size of a Sidon set contained in a random set of integers.

Motivated by this literature, we consider threshold functions for the existence of certain substructures in random subsets of vector spaces over finite fields. 


\subsection{Notation and our main results}

Let $\mathbb{F}_{q}$ denote the finite field with $q$ elements, where $q$ is a prime power, and let $\mathbb{F}_{q}^{n}$ be the $n$-dimensional vector space over this field. When $q$ is fixed and $n$ is large, the set $\mathbb{F}_{q}^{n}$ is known as the finite field model, and was suggested by Green [23] as a toy model for testing proof techniques in additive number theory. This gave a simplified setting which retained key features of the original problem. See the survey of Wolf [38] for more details.

Given $0<\delta<1$, let $E$ be a random subset of $\mathbb{F}_{q}^{n}$ chosen as follows: put $x \in E$ with probability $\delta$ and $x \notin E$ with probability $1-\delta$, independently for all $x \in \mathbb{F}_{q}^{n}$. Denote the resulting probability space by $\Omega\left(\mathbb{F}_{q}^{n}, \delta\right)$. The model $\Omega\left(\mathbb{F}_{q}^{n}, \delta\right)$ is closely related to the Gilbert random graph model $G(n, p)$, see for example [18], and to random subsets of $[N]=\{1,2, \ldots, N\}$. When working with finite fields, it is common to use $p$ to denote a prime number, rather than a probability. This is why we use $\delta$ to denote the probability parameter in our model.

Standard asymptotic notation is given at the start of Section 2. All asymptotics are as $q+n \rightarrow \infty$ unless otherwise stated.

Let $P$ be a combinatorial property and $E \in \Omega\left(\mathbb{F}_{q}^{n}, \delta\right)$. Say that $P$ is a monotone increasing property if the property is inherited by supersets, and a monotone decreasing property if it is inherited by subsets. Then $t(n, q)$ is a (coarse) threshold function for the monotone increasing property $P$ if the following holds:

(i) if $\delta=o(t(n, q))$ then $\mathbb{P}(E$ satisfies property $P) \rightarrow 0$, and

(ii) if $\delta=\omega(t(n, q))$ then $\mathbb{P}(E$ satisfies property $P) \rightarrow 1$.

If $P$ is a decreasing properties then a coarse threshold is defined by applying the above definition to the negation of $P$. Most properties we consider involve containment of a given substructure, so they are increasing properties. Bollobás and Thomason [6] proved that every non-trivial monotone increasing property of sets has a coarse threshold.

Let $\mathcal{A}=\mathcal{A}_{a}$ be a family of subsets of $\mathbb{F}_{q}^{n}$ such that each element of $\mathcal{A}$ contains $a$ vectors, where $a$ is a fixed constant. Let $X=X_{\mathcal{A}}(E)$ be the random variable which counts the elements of $\mathcal{A}$ in the random set $E$. That is,

$$
X=X_{\mathcal{A}}(E)=\sum_{T \in \mathcal{A}} \mathbf{1}_{E}(T)
$$

where $\mathbf{1}_{E}(T)$ equals 1 if $T \subseteq E$ and equals 0 otherwise. We write $|S|$ to denote the cardinality of a set $S$. By linearity of expectation we have

$$
\mathbb{E}(X)=|\mathcal{A}| \delta^{a}=: \lambda_{\mathcal{A}}
$$

Let $\operatorname{Po}(\mu)$ denote the Poisson distribution with mean $\mu$, and write $X \stackrel{\mathrm{d}}{\rightarrow} \operatorname{Po}(\mu)$ if $X$ tends in distribution to $\operatorname{Po}(\mu)$.

Our main contribution is the following general theorem which can be used to locate a coarse threshold for the property "contains an element of $\mathcal{A}$ ", and to prove that that 
the number of patterns in $\mathcal{A}$ contained in $E$ has a Poisson distribution when its expected value tends to a finite limit.

Theorem 1.1. Suppose there exist integer constants $b>0, c \geq 0$ such that $|\mathcal{A}|=$ $\Theta\left(q^{b n-c}\right)$. Assume that for any set $S$ of $k$ distinct points in $\mathbb{F}_{q}^{n}$, the number of elements of $\mathcal{A}$ which contain $S$ is

$$
\begin{cases}O\left(q^{(b-k) n-c}\right) & \text { if } 1 \leq k \leq b-1 \\ O(1) & \text { otherwise. }\end{cases}
$$

Then the event "contains an element of $\mathcal{A}$ " has a coarse threshold function

$$
t(n, q)=q^{(c-b n) / a} .
$$

(Above, asymptotics are as $q+n \rightarrow \infty$.) Furthermore, if $\mathbb{E}(X) \rightarrow \lambda$ for some constant $\lambda>0$ then $X \stackrel{d}{\rightarrow} \operatorname{Po}(\lambda)$, where asymptotics are as

$$
\begin{cases}q+n \rightarrow \infty & \text { if } b<a, \\ q \rightarrow \infty & \text { if } b=a \text { and } c>0 .\end{cases}
$$

This framework only applies to patterns with a high degree of regularity, as captured by the condition (3). To generalise Theorem 1.1, it may be necessary to adapt the concepts of balanced and strictly balanced graphs (discussed in the introduction) to consider the densest substructures in a given pattern, in some sense. This is left for future work, see Section 5.3.

We will illustrate Theorem 1.1 by applying it to some well-studied patterns described below. The parameter which tends to infinity is specified for each pattern.

\section{Definition 1.2.}

- For fixed $k \geq 3$, a non-trivial $k$-term arithmetic progression consists of $k$ distinct vectors

$$
x, x+v, \ldots, x+(k-1) v
$$

of $\mathbb{F}_{q}^{n}$. Asymptotics are as $q+n$ tends to infinity.

- A sum consists of vectors $x, y, z$ (not necessarily distinct) such that $x+y=z$.

- A non-trivial parallelogram consists of four distinct vectors $x_{1}, x_{2}, x_{3}, x_{4}$ of $\mathbb{F}_{q}^{n}$ such that

$$
x_{\sigma(1)}-x_{\sigma(2)}=x_{\sigma(4)}-x_{\sigma(3)}
$$

for some permutation $\sigma$. Asymptotics are as $q+n$ tends to infinity.

- A non-trivial right triangle consists of three distinct vectors $x_{1}, x_{2}, x_{3}$ of $\mathbb{F}_{q}^{n}$ such that

$$
\left(x_{\sigma(2)}-x_{\sigma(1)}\right) \cdot\left(x_{\sigma(3)}-x_{\sigma(1)}\right)=0
$$

for some permutation $\sigma$. Asymptotics for the threshold result are as $q+n$ tends to infinity. For the Poisson limit result we take asymptotics as $q \rightarrow \infty$, with $n \geq 2$. (Here $n$ may be bounded or growing.) 
- An $m$-dimensional plane is a translation of an $m$-dimensional subspace, for any $m \in\{1,2, \ldots, n-1\}$, where $n \geq 2$. Each $m$-dimensional plane contains $q^{m}$ vectors. Asymptotics are as $n$ tends to infinity, with $q$ and $m$ fixed.

In the definition of $k$-APs, parallelograms and right triangles, "non-trivial" means that the vectors in the pattern are distinct. For ease of exposition we sometimes omit the word "non-trivial" when referring to these patterns.

Observe that (non-trivial) right triangles cannot be expressed as the solution of a system of linear equations. Similarly, $m$-dimensional planes do not have an analogue in the setting of random subsets of integers.

Furstenberg and Katznelson [20] proved that there is a function $Q(\varepsilon, q, m)$ defined for all positive integers $m$, prime powers $q$ and $\varepsilon>0$, such that if $n>Q(\varepsilon, q)$ and $S \subseteq \mathbb{F}_{q}^{n}$ satisfies $|S| \geq \varepsilon q^{n}$ then $S$ contains an $m$-dimensional affine subspace. One of our applications, below, is a threshold result for the appearance of $m$-dimensional affine planes in random subgraphs of $\mathbb{F}_{q}^{n}$.

Theorem 1.3. Let $\mathcal{A}$ be one of the collections of patterns defined in Definition 1.2. The functions

$$
t(n, q)= \begin{cases}q^{-2 n / k} & k \text {-APs } \\ q^{-2 n / 3} & \text { sums } \\ q^{-3 n / 4} & \text { parallelograms } \\ q^{-n+1 / 3} & \text { right triangles } \\ q^{-(m+1) n / q^{m}} & m \text {-dimensional planes }\end{cases}
$$

are threshold functions for the property " $E$ contains an element of $\mathcal{A}$ ". Furthermore, if $\lambda_{\mathcal{A}} \rightarrow \lambda$ where $\lambda \in(0, \infty)$ then $X \stackrel{d}{\rightarrow} \operatorname{Po}(\lambda)$, where $X$ is the number of elements of $\mathcal{A}$ contained in the random set $E$, and $\lambda_{\mathcal{A}}$ is defined in (2). All asymptotics are taken with the respect to the limits described in Definition 1.2.

A set $A \subseteq \mathbb{F}_{q}^{n}$ is sum-free if there are no elements $x, y, z \in \mathbb{F}_{q}^{n}$ with $x+y=z$. A Sidon set in $\mathbb{F}_{q}^{n}$ is a subset $A \subseteq \mathbb{F}_{q}^{n}$ such that sums of elements of $A$ are unique, up to permutation of the summands. That is, in a Sidon set $A$, if $x+y=z+w$ for $x, y, z, w \in A$ then $(x, y)=(z, w)$ or $(x, y)=(w, z)$. The next two results follow easily from Theorem 1.3 (indeed, Corollary 1.4 is immediate).

Corollary 1.4. The function $t(n, q)=q^{-2 n / 3}$ is a coarse threshold for the (decreasing) property that $E$ is sum-free, as $q+n \rightarrow \infty$.

Corollary 1.5. The function $t(n, q)=q^{-3 n / 4}$ is a coarse threshold for the (decreasing) property that $E$ is a Sidon set, as $q+n \rightarrow \infty$.

Proof. Observe that $E$ is a Sidon set if and only if it contains no non-trivial parallelograms and no non-trivial 3-APs. If $\delta=o\left(q^{-3 n / 4}\right)$ then also $\delta=o\left(q^{-2 n / 3}\right)$, so the probability that $E$ contains a non-trivial parallelogram or non-trivial 3-AP tends to zero. That is, the probability that $E$ is a Sidon set tends to 1 when $\delta=o\left(q^{-3 n / 4}\right)$. Conversely, if $\delta=\omega\left(q^{-3 n / 4}\right)$ then $E$ contains a non-trivial parallelogram with probability which tends to 1 , so the probability that $E$ is a Sidon set tends to zero. 
We note that there are many other interesting patterns in $\mathbb{F}_{q}^{n}$, such as Kakeya sets [12] and Furstenberg sets [13].

Our proofs use standard arguments, namely the first and second moment method and the method of moments.

We will show that if $\delta=o(t(n, q))$ then $\mathbb{E}\left(X_{\mathcal{A}}\right)$ tends to zero. The negative side of the threshold result then follows directly from Markov's inequality (first moment method), since

$$
\mathbb{P}\left(X_{\mathcal{A}} \geq 1\right) \leq \mathbb{E}\left(X_{\mathcal{A}}\right) .
$$

The positive side of the threshold result is completed using the second moment method. Finally, the proof of Theorem 1.3 is completed by studying the higher order moments of the random variable $X_{\mathcal{A}}$ and applying the method of moments.

The rest of the paper is organised as follows. First we finish this section by discussing some other related work. In Section 2 we introduce some notation, then state conditions which are sufficient for the second moment and method of moments arguments. We prove our general theorem, Theorem 1.1, in Section 3. Then in Section 4 we apply Theorem 1.1 to prove Theorem 1.3 for $k$-APs, sums, right triangles, and parallelograms. The case of $m$-dimensional planes requires special care and is treated separately in Section 4.1. Section 5 contains some further remarks, including applications to extremal problems and directions for future research.

\subsection{Other related work}

There are Ramsey-theoretic versions of the problem of existence of a given substructure in a random combinatorial object. For example, graph $\Gamma$ is $(G, k)$-Ramsey if there is a monochromatic copy of $G$ in any colouring of the edges of $\Gamma$ with $k$ colours. Rödl and Ruciński [30] proved that if $G$ is not a forest of stars and paths then there are constants $c, C>0$ such that

$$
\lim _{n \rightarrow \infty} \operatorname{Pr}(G(n, p) \text { is }(G, k) \text {-Ramsey })= \begin{cases}0 & \text { if } p<c n^{-1 / m_{2}(G)} \\ 1 & \text { if } p>C n^{-1 / m_{2}(G)}\end{cases}
$$

where

$$
m_{2}(G)=\max \left\{\frac{|E(H)|-1}{|H|-2}: H \subseteq G\right\} .
$$

Let $[n]_{p}$ denote the random subset of $[n]$, where each element is chosen independent with probability $p$. Schur's Theorem says that for every $r \geq 2$ and for $n$ sufficiently large, in any $r$-colouring of $[n]$ there must be a monochromatic solution to $x+y=z$. A solution with $x \neq y$ is called a Schur triple. Graham, Rödl and Ruciński [21] proved that the threshold for any 2-colouring of $[n]_{p}$ to have a monochromatic Schur triple is $p=n^{-1 / 2}$, and gave a lower bound on the number of Schur triples when $p n^{1 / 2} \rightarrow \infty$.

Given an $\ell \times k$ matrix $A=\left(a_{i j}\right)$ of integers, say that $A$ is partition regular if for any finite colouring of $\mathbb{N}$ there is a monochromatic solution to the system of linear 
equations $A \boldsymbol{x}=\mathbf{0}$. We may assume that $A$ has full rank and that it is irredundant, which means that for each $i \neq j$ there is a solution $\boldsymbol{x}=\left(x_{1}, \ldots, x_{k}\right)$ with $x_{i} \neq x_{j}$. An irredundant matrix $A$ is density regular if $\boldsymbol{x}=(1,1, \ldots, 1)$ is a solution of $A \boldsymbol{x}=\mathbf{0}$. Rödl and Ruciński [31] proved a threshold result for the existence of monochromatic solutions to $A \boldsymbol{x}=\mathbf{0}$ in $[n]_{p}$, given any colouring of $[n]$ using $r$ colours, when $A$ is an irredundant, density regular integer matrix $A$. The threshold is determined by a parameter $m_{A}$ which is a maximum over all sets of columns of a function involving the rank of the submatrix induced by the chosen columns. They conjectured that the same threshold result should hold for any partition regular system of equations (without the density-regular condition).

Similarly, many researchers have considered "sparse random" versions of extremal problems. Kohayakawa, Łuczak and Ruciński [27] studied 3-APs in $[n]_{p}$. Given $0<\alpha<$ 1 , a subset $A \subset[n]$ is called $\alpha-3 A P$ if every subset $F \subseteq A$ with $|F| \geq \alpha|A|$ contains a three-term arithmetic progression. Kohayakawa et al. [27] found a threshold for the probability that $[n]_{p}$ is $\alpha-3 \mathrm{AP}$, using the Szemerédi regularity lemma.

Conlon and Gowers [11], Schacht [35] and Friedgut, Rödl and Schacht [19] presented powerful methods for attacking sparse random versions of Ramsey-theoretic and extremal combinatorial questions. Several conjectured results were established in these works. In particular, Conlon and Gowers [11] and independently, Schacht [35] determined the threshold for Szemerédi's theorem in random subsets of the integers, and Friedgut et al [19] proved a threshold result for Rado's theorem on solutions of partition regular systems of equations in random subsets of integers, proving the conjecture made by Rödl and Ruciński [31].

Recently there has been a growing interest in studying finite field versions of some classical problems arising from Euclidean spaces. For instance, there are finite field Besicovitch-Kakeya sets [12, 23, 39]; finite field Erdős-Falconer distance problem [24] and finite fields version of restriction problems of Fourier analysis [29]. This was an additional motivation for our study. From another point of view, $\Omega\left(\mathbb{F}_{q}^{n}, \delta\right)$ can be considered as a discrete version of fractal percolation (Mandelbrot percolation), see [17, Chapter 15]. For some applications of $\Omega\left(\mathbb{F}_{q}^{n}, \delta\right)$ to deterministic problems, we refer to [2, Theorem 5.2] and [9] for finding subsets of $\mathbb{F}_{q}^{n}$ with small Fourier coefficients, and [10, Theorems 1.5 and 1.6] for finding sets without exceptional projections.

\section{Preliminaries}

The following standard asymptotic notation will be used, assuming that asymptotics are taken with respect to a parameter $N$. Write "a.a.s." to mean asymptotically almost surely, which means that the property holds with probability which tends to 1 . We write $f=O(g)$ if there is a positive constant $C$ such that $|f(N)| \leq C|g(N)|$ for all $N$ sufficiently large, and write $f=\Omega(g)$ if $g=O(f)$. If $f=O(g)$ and $f=\Omega(g)$ then $f=\Theta(g)$. We write $f=o(g)$ if $f(N) / g(N) \rightarrow 0$, and write $f=\omega(g)$ if $g=o(f)$. Finally, $f \sim g$ means that $f=g(1+o(1))$.

We also use $f=O_{L}(g)$ to mean that $|f(N)| \leq C|g(N)|$ for all $N$ sufficiently large, 
where $L$ is a list of parameters and $C$ is a positive constant which depends on $L$.

The parameter $\lambda$ always denotes a fixed positive real number.

Recall that $\mathcal{A}=\mathcal{A}_{a}$ is a family of subsets of $\mathbb{F}_{q}^{n}$ such that every element of $\mathcal{A}$ contains $a$ points. Our asymptotics are with respect to some function $N=N(q, n)$ which tends to infinity. In the calculations for the second moment, we consider intersections of pairs of elements of $\mathcal{A}$. For $k=0,1, \ldots, a$, define

$$
I_{k}=I_{\mathcal{A}, k}=\left\{\left(T, T^{\prime}\right): T, T^{\prime} \in \mathcal{A},\left|T \cap T^{\prime}\right|=k\right\} .
$$

Let $Y=Y_{\mathcal{A}}$ be the random variable which counts pairs $\left(T, T^{\prime}\right)$ of distinct elements of $\mathcal{A}$ with non-empty intersection such that both $T, T^{\prime}$ are contained in the random set $E$. That is,

$$
Y=Y_{\mathcal{A}}=\sum_{\substack{T, T^{\prime} \in \mathcal{A} \\ 1 \leq\left|T \cap T^{\prime}\right| \leq a-1}} \mathbf{1}_{E}\left(T \cup T^{\prime}\right)=\sum_{k=1}^{a-1} \sum_{\left(T, T^{\prime}\right) \in I_{k}} \mathbf{1}_{E}\left(T \cup T^{\prime}\right)
$$

Recall the definition of $X$ from (1).

Lemma 2.1 (Second moment). Suppose that $\mathbb{E}(X)=|\mathcal{A}| \delta^{a} \rightarrow \infty$ with respect to some $N=N(q, n) \rightarrow \infty$, and that

(C1) $\left|I_{0}\right| \sim|\mathcal{A}|^{2}$

(C2) $\mathbb{E}(Y)=o\left(\mathbb{E}(X)^{2}\right)$.

Then a.a.s. E contains some element of $\mathcal{A}$.

Proof. Observe that $\left|I_{a}\right|=|\mathcal{A}|$, and hence that $\left|I_{a}\right| \delta^{a}=\mathbb{E}(X)=o\left(\mathbb{E}(X)^{2}\right)$. Therefore, using $(\mathrm{C} 1)$ and $(\mathrm{C} 2)$ we have

$$
\begin{aligned}
\mathbb{E}\left(X^{2}\right) & =\mathbb{E}\left(\sum_{\left(T, T^{\prime}\right) \in \mathcal{A} \times \mathcal{A}} \mathbf{1}_{E}(T) \mathbf{1}_{E}\left(T^{\prime}\right)\right) \\
& =\left|I_{0}\right| \delta^{2 a}+\left|I_{a}\right| \delta^{a}+\mathbb{E}(Y) \\
& \sim \mathbb{E}(X)^{2}
\end{aligned}
$$

Hence, by the Paley-Zygmund inequality,

$$
\mathbb{P}(X>0) \geq \frac{\mathbb{E}(X)^{2}}{\mathbb{E}\left(X^{2}\right)} \rightarrow 1
$$

completing the proof.

Now suppose that $\mathbb{E}(X) \rightarrow \lambda$ where $\lambda \in(0, \infty)$. We consider the factorial moment $\mathbb{E}\left((X)_{r}\right)$ of the random variable $X$ for positive integers $r$. Here

$$
(X)_{r}:=X(X-1) \ldots(X-r+1) .
$$

We will show that $\mathbb{E}\left((X)_{r}\right) \rightarrow \lambda^{r}$ for all $r \in \mathbb{N}$. From this, the method of moments implies that $X$ converges in distribution to a Poisson distribution with parameter $\lambda$. 
Lemma 2.2. (Bollobas [5, Theorem 1.22]) Let $\mu$ be a positive real constant. If for all $r \in \mathbb{N}$ the factorial moment $\mathbb{E}\left((X)_{r}\right) \rightarrow \mu^{r}$ with respect to some $N=N(q, n) \rightarrow \infty$, then $X$ converges in distribution to a Poisson distribution with mean $\mu$.

Recall the definition of $I_{k}$ from (6) and define

$$
I_{\geq 1}=\bigcup_{k=1}^{a} I_{k} .
$$

The following lemma can be used to check that the conditions of Lemma 2.2 hold.

Lemma 2.3 (Higher moments). Assume that $|\mathcal{A}| \rightarrow \infty$ with respect to some $N=$ $N(q, n) \rightarrow \infty$ and let $X=X_{\mathcal{A}}$ be as defined in (1). Suppose that $\mathbb{E}(X) \rightarrow \lambda$ where $\lambda$ is a positive real number, and that the following conditions hold:

(C1) $\left|I_{0}\right| \sim|\mathcal{A}|^{2}$

$(\mathrm{C} 2) \mathbb{E}(Y)=o\left(\mathbb{E}(X)^{2}\right)$;

(C3) $\mathbb{E}\left(X^{r}\right)=O_{r}(1)$ for all fixed integers $r \geq 2$.

Then $\mathbb{E}\left((X)_{r}\right) \rightarrow \lambda^{r}$ for all $r \in \mathbb{N}$.

Proof. For any integer $r \geq 2$, define

$$
(\mathcal{A})_{r}:=\left\{\left(T_{1}, \ldots, T_{r}\right) \in \mathcal{A}^{r}: T_{1}, \ldots, T_{r} \text { are pairwise distinct }\right\} .
$$

Let

$$
\Gamma_{0}^{(r)}=\left\{\left(T_{1}, \ldots, T_{r}\right) \in(\mathcal{A})_{r}: T_{1}, \ldots, T_{r} \text { are pairwise disjoint }\right\},
$$

and write $\Gamma_{\geq 1}^{(r)}=(\mathcal{A})_{r} \backslash \Gamma_{0}^{(r)}$. Observe that

$$
\left|\Gamma_{\geq 1}^{(r)}\right| \leq\left(\begin{array}{c}
r \\
2
\end{array}\right)\left|I_{\geq 1}\right||\mathcal{A}|^{r-2} .
$$

Condition (C1) implies that $\left|I_{\geq 1}\right|=o\left(|\mathcal{A}|^{2}\right)$, and hence

$$
\left|\Gamma_{\geq 1}^{(r)}\right|=o\left(|\mathcal{A}|^{r}\right) .
$$

It follows that

$$
\left|\Gamma_{0}^{(r)}\right| \sim\left|(\mathcal{A})_{r}\right|
$$

Write the random variable $(X)_{r}$ as

$$
(X)_{r}=\sum_{t \in(\mathcal{A})_{r}} \mathbf{1}_{E}(t)=\sum_{t \in \Gamma_{0}^{(r)}} \mathbf{1}_{E}(t)+\sum_{t \in \Gamma_{\geq 1}^{(r)}} \mathbf{1}_{E}(t) .
$$

Applying the estimate (8) and the assumption that $|\mathcal{A}| \rightarrow \infty$, we obtain

$$
\mathbb{E}\left(\sum_{t \in \Gamma_{0}^{(r)}} \mathbf{1}_{E}(t)\right)=\left|\Gamma_{0}^{(r)}\right| \delta^{a r} \sim\left|(\mathcal{A})_{r}\right| \delta^{a r} \sim \lambda^{r}
$$


It remains to prove that $\mathbb{E}\left(\sum_{t \in \Gamma_{\geq 1}^{(r)}} \mathbf{1}_{E}(t)\right)=o(1)$.

Recall that $Y$, defined in (7), counts pairs of distinct elements of $\mathcal{A}$ with nonempty intersection which are contained in $E$. If $Y=0$ with probability one then $\sum_{t \in \Gamma_{\geq 1}^{(r)}} \mathbf{1}_{E}(t)=0$ with probability one. Hence the result holds in this trivial case. For the remainder of the proof, suppose that $Y>0$ with positive probability. Then $\mathbb{E}(Y)>0$, so we may define

$$
M=M_{Y, r}:=\mathbb{E}(Y)^{-\frac{1}{2 r}} .
$$

Write

$$
\sum_{t \in \Gamma_{\geq 1}^{(r)}} \mathbf{1}_{E}(t)=\mathbf{1}_{(X<M)} \sum_{t \in \Gamma_{\geq 1}^{(r)}} \mathbf{1}_{E}(t)+\mathbf{1}_{(X \geq M)} \sum_{t \in \Gamma_{\geq 1}^{(r)}} \mathbf{1}_{E}(t) .
$$

Note that for any $t=\left(T_{1}, \ldots, T_{r}\right) \in \Gamma_{\geq 1}^{(r)}$ there exists $1 \leq i<j \leq r$ with $\left|T_{i} \cap T_{j}\right| \geq 1$. Such pairs which are contained in $E$ are counted by $Y$, and there are $\left(\begin{array}{l}r \\ 2\end{array}\right)$ choices for $(i, j)$. Furthermore, when $X<M$ then there are at most $M^{r-2}$ choices for the other entries of $t$. Therefore, using the union bound,

$$
\mathbf{1}_{(X<M)} \sum_{t \in \Gamma_{\geq 1}^{(r)}} \mathbf{1}_{E}(t) \leq\left(\begin{array}{l}
r \\
2
\end{array}\right) Y M^{r-2} .
$$

By definition of $M$ and by assumption (C2), we have $\mathbb{E}(Y)=o\left(\mathbb{E}(X)^{2}\right) \rightarrow 0$ and hence

$$
\mathbb{E}\left(\mathbf{1}_{(X<M)} \sum_{t \in \Gamma_{\geq 1}^{(r)}} \mathbf{1}_{E}(t)\right) \leq\left(\begin{array}{l}
r \\
2
\end{array}\right) \mathbb{E}\left(Y M^{r-2}\right)=\left(\begin{array}{l}
r \\
2
\end{array}\right) \mathbb{E}(Y)^{\frac{r+2}{2 r}} \rightarrow 0 .
$$

So the first summand in (10) is vanishing.

For the second summand in (10), observe that $\sum_{t \in \Gamma_{>1}^{(r)}} \mathbf{1}_{E}(t) \leq X^{r}$. Using this and the Cauchy-Schwarz inequality (in the form $|\mathbb{E}(Z W)|^{2} \leq \mathbb{E}\left(Z^{2}\right) \mathbb{E}\left(W^{2}\right)$ for random variables $Z, W)$, we obtain

$$
\mathbb{E}\left(\mathbf{1}_{(X \geq M)} \sum_{t \in \Gamma_{\geq 1}^{(r)}} \mathbf{1}_{E}(t)\right) \leq \mathbb{E}\left(\mathbf{1}_{(X \geq M)} X^{r}\right) \leq \mathbb{E}\left(\mathbf{1}_{(X \geq M)}\right)^{1 / 2} \mathbb{E}\left(X^{2 r}\right)^{1 / 2} .
$$

Now assumption (C3) says that $\mathbb{E}\left(X^{2 r}\right)=O_{r}(1)$, and

$$
\mathbb{E}\left(\mathbf{1}_{(X \geq M)}\right)=\mathbb{P}(X \geq M) \leq \frac{\mathbb{E}(X)}{M}=\mathbb{E}(X) \mathbb{E}(Y)^{\frac{1}{2 r}} \rightarrow 0
$$

using Markov's inequality, the definition of $M$ and the fact that $\mathbb{E}(Y) \rightarrow 0$, which follows from assumption (C2). Hence the second term of (10) satisfies

$$
\mathbb{E}\left(\mathbf{1}_{(X \geq M)} \sum_{t \in \Gamma_{\geq 1}^{(r)}} \mathbf{1}_{E}(t)\right)=O_{r}(1)\left(\mathbb{E}(X) \mathbb{E}(Y)^{\frac{1}{2 r}}\right)^{1 / 2} \rightarrow 0 .
$$

Combining (11) and (12) shows that (10) is vanishing, and together with (9), this completes the proof. 


\section{Proof of the general theorem}

In this section we prove Theorem 1.1. Recall that every element of $\mathcal{A}$ contains $a$ vectors, and that $a$ is a fixed positive integer.

Proof of Theorem 1.1. Since $\mathcal{A} \subseteq \mathbb{F}_{q}^{n}$ it follows immediately that $b \leq a$. Asymptotics are as $q+n \rightarrow \infty$ unless otherwise specified.

By definition of $t(n, q)$, if $\delta=o(t(n, q))$ then

$$
\mathbb{E}(X)=|\mathcal{A}| \delta^{a}=|\mathcal{A}| o\left(q^{c-b n}\right)=o(1) .
$$

This establishes the negative side of the threshold result.

Next we estimate the cardinality of the set $I_{k}$ defined in (6). For any $\left(T, T^{\prime}\right) \in I_{k}$ with $k \in\{1, \ldots, b-1\}$, the sets $T$ and $T^{\prime}$ intersect in $k$ points of $\mathbb{F}_{q}^{n}$. As there are $O\left(q^{k n}\right)$ choices for this intersection, it follows that

$$
\left|I_{k}\right|=O\left(q^{k n}\right) O\left(q^{2(b-k) n-2 c}\right)=|\mathcal{A}|^{2} O\left(q^{-k n}\right) .
$$

If $k \in\{b, \ldots, a-1\}$ then $k$ points determine $O(1)$ elements of $\mathcal{A}$, so $\left|I_{k}\right| \leq|\mathcal{A}|\left(\begin{array}{l}a \\ k\end{array}\right) O(1)^{2}$. Hence, as $a$ is a fixed constant,

$$
\left|I_{k}\right|= \begin{cases}|\mathcal{A}|^{2} O\left(q^{-k n}\right) & \text { if } 1 \leq k \leq b-1 \\ |\mathcal{A}|^{2} O\left(q^{-b n+c}\right) & \text { otherwise }\end{cases}
$$

It follows that

$$
\left|I_{0}\right|=|A|^{2}-|A|-\sum_{k=1}^{a-1}\left|I_{k}\right|=|A|^{2}\left(1-O\left(q^{-n+c}\right)\right) \sim|A|^{2},
$$

and condition (C1) holds.

Recall the random variables $X$ and $Y$ defined in (1) and (7), respectively. Observe that

$$
\begin{aligned}
\mathbb{E}(Y) & =\sum_{k=1}^{a-1}\left|I_{k}\right| \delta^{2 a-k} \\
& =|A|^{2} \delta^{2 a}\left(\sum_{k=1}^{b-1} q^{-n k} \delta^{-k}+\sum_{k=b}^{a-1} q^{-b n+c} \delta^{-k}\right) .
\end{aligned}
$$

Note that if $a=b$ then the summation from $b$ to $a-1$ vanishes, leaving only the first summation. Next, $\mathbb{E}(X)=\Theta\left(q^{b n-c}\right) \delta^{a}$, and hence

$$
q^{n} \delta=\Theta\left(q^{n\left(1-\frac{b}{a}\right)+\frac{c}{a}} \mathbb{E}(X)^{\frac{1}{a}}\right) .
$$

The expression (14) tends to infinity if $\mathbb{E}(X) \rightarrow \infty$ (whether $q$ is fixed or tends to infinity). If $\mathbb{E}(X) \rightarrow \lambda$ for some constant $\lambda$, then the expression in (14) tends to infinity 
whenever $b<a$, or when $b=a, c>0$ and $q \rightarrow \infty$. Furthermore, for $k=b, \ldots, a$, we claim that

$$
q^{b n-c} \delta^{k}=\Theta(\mathbb{E}(X)) \delta^{k-a} \rightarrow \infty
$$

when $\mathbb{E}(X) \rightarrow \infty$ or when $\mathbb{E}(X) \rightarrow \lambda$ for some fixed positive $\lambda$. This is clear when $\mathbb{E}(X) \rightarrow \infty$, as $k<a$; on the other hand, if $\mathbb{E}(X) \rightarrow \lambda$ for some constant $\lambda$ then we must have $\delta=o(1)$, so $\mathbb{E}(X) \delta^{k-a}=\Theta\left(\delta^{k-a}\right) \rightarrow \infty$.

Using the assumptions of Theorem 1.1, we conclude that $\mathbb{E}(Y)=o\left(\mathbb{E}(X)^{2}\right)$ if one of the following conditions holds:

- $\mathbb{E}(X) \rightarrow \infty$,

- $b<a$ and $\mathbb{E}(X) \rightarrow \lambda$ for some constant $\lambda$, and $q+n \rightarrow \infty$,

- $b=a, c>0$ and $\mathbb{E}(X) \rightarrow \lambda$ for some constant $\lambda$, and $q \rightarrow \infty$.

So (C2) holds when one of the above conditions holds, under the assumptions of Theorem 1.1.

If $\delta=\omega(t(n, q))$ then $\mathbb{E}(X) \rightarrow \infty$, and the above arguments show that $(\mathrm{C} 1)$ and (C2) hold as $q+n \rightarrow \infty$. By Lemma 2.1, we conclude that a.a.s. $E$ contains some element of $\mathcal{A}$. This completes the proof that $t(n, q)$ is a coarse threshold function for $\mathcal{A}$.

For the remainder of the proof we assume that $\mathbb{E}(X) \rightarrow \lambda$ for some fixed positive $\lambda$. If $b=a$ then we additionally assume that $q \rightarrow \infty$. We will establish (C3) by proving that $\mathbb{E}\left(X^{r}\right)=O_{r}(1)$, by induction on $r$. The case $r=1$ holds by assumption. Now we suppose that $\mathbb{E}\left(X^{r}\right) \leq C_{r}$ holds for some $r \geq 1$. Observe that

$$
\begin{aligned}
\mathbb{E}\left(X^{r}\right) & =\mathbb{E}\left(\sum_{\left(T_{i_{1}}, \ldots, T_{i_{r}}\right) \in \mathcal{A}^{r}} \mathbf{1}_{E}\left(T_{i_{1}}\right) \ldots \mathbf{1}_{E}\left(T_{i_{r}}\right)\right) \\
& =\sum_{\left(T_{i_{1}}, \ldots, T_{i_{r}}\right) \in \mathcal{A}^{r}} \mathbb{P}\left(\cup_{\ell=1}^{r} T_{i_{\ell}} \subset E\right) .
\end{aligned}
$$

Moreover, by the law of total probability,

$$
\begin{aligned}
\mathbb{E}\left(X^{r+1}\right) & =\sum_{\left(T_{i_{1}}, \ldots, T_{i_{r+1}}\right) \in \mathcal{A}^{r+1}} \mathbb{P}\left(\cup_{\ell=1}^{r+1} T_{i_{\ell}} \subset E\right) \\
& =\sum_{\left(T_{i_{1}}, \ldots, T_{i_{r}}\right) \in \mathcal{A}^{r}} \sum_{T_{i_{r+1}} \in \mathcal{A}} \mathbb{P}\left(T_{i_{r+1}} \subset E \mid \cup_{\ell=1}^{r} T_{i_{\ell}} \subset E\right) \mathbb{P}\left(\cup_{\ell=1}^{r} T_{i_{\ell}} \subset E\right) .
\end{aligned}
$$

Together with (15), we conclude that it is sufficient to prove that there exists positive constant $\widehat{C}_{r}$ such that for any $T_{1}, \ldots, T_{r} \in \mathcal{A}$,

$$
\sum_{T \in \mathcal{A}} \mathbb{P}\left(T \subset E \mid \cup_{\ell=1}^{r} T_{\ell} \subset E\right) \leq \widehat{C}_{r} .
$$


Indeed suppose that (16) is true, then the above argument implies

$$
\mathbb{E}\left(X^{r+1}\right) \leq \widehat{C}_{r} C_{r}=O_{r}(1)
$$

and we may take $C_{r+1}=\widehat{C}_{r} C_{r}$.

We now turn to the proof of (16). Let $B=\bigcup_{i=1}^{r} T_{i}$. For $0 \leq k \leq a$, define

$$
J_{k}=\{T \in \mathcal{A}:|T \cap B|=k\} .
$$

It follows from (13) that

$$
\left|J_{k}\right|= \begin{cases}O_{r}\left(q^{(b-k) n-c}\right) & \text { if } 0 \leq k \leq b-1 \\ O_{r}(1) & \text { otherwise }\end{cases}
$$

We know that $q^{n} \delta \rightarrow \infty$, as proved below (14), requiring the additional assumption that $q \rightarrow \infty$ when $b=a$. Therefore

$$
\begin{aligned}
\sum_{T \in \mathcal{A}} \mathbb{P}\left(T \subset E \mid \cup_{\ell=1}^{r} T_{\ell} \subset E\right) & =\sum_{k=0}^{a}\left|J_{k}\right| \delta^{a-k} \\
& =\sum_{k=0}^{b-1} \lambda O\left(q^{-k n} \delta^{-k}\right)+\sum_{k=b}^{a} O(1) \delta^{a-k} \\
& =O_{\lambda, r}(1) .
\end{aligned}
$$

This shows that (C3) holds, and hence by Lemma 2.3 we conclude that $X \stackrel{\mathrm{d}}{\rightarrow} \operatorname{Po}(\lambda)$, completing the proof.

What can we say about the distribution of $X$ in the case that $\mathbb{E}(X) \rightarrow \lambda$ for some positive constant $\lambda$, but $b=a$ and $q$ is fixed? From (14) we see that

$$
q^{n} \delta=\Theta\left(q^{c / a} \mathbb{E}(X)^{1 / a}\right)=\Theta(1),
$$

which implies that $\mathbb{E}(Y)=\Theta\left(\mathbb{E}(X)^{2}\right)$. Thus condition (C2) fails, and the argument of Lemma 2.3 breaks down, as it is no longer true that most pairs of patterns in $E$ are disjoint. It is possible that in this case $X$ has a normal distribution. We leave this as an open question.

\section{Applications}

The desired results for almost all of the patterns described in Definition 1.2 follow immediately from Theorem 1.1. The argument for $m$-dimensional subspaces is slightly more delicate and is given in Section 4.1 below.

Corollary 4.1. Theorem 1.3 holds for $k$-APs, sums, parallelograms and right triangles. 


\begin{tabular}{|c|c|c|c|}
\hline $\mathcal{A}_{a}$ & $a$ & $b$ & $c$ \\
\hline$k$-APs & $k$ & 2 & 0 \\
\hline sums & 3 & 2 & 0 \\
\hline parallelograms & 4 & 3 & 0 \\
\hline right triangles & 3 & 3 & 1 \\
\hline
\end{tabular}

Table 1: Parameter values for $k$-APs, sums, parallelograms and right triangles

Proof. It suffices to prove that the conditions of Theorem 1.1 hold with the parameters given in Table 1, noting that the asymptotic assumptions of Theorem 1.1 match those specified in Definition 1.2.

We give the proof for right triangles only: the proof for the other patterns follow similarly. Let $\mathcal{A}$ be the set of all right triangles in $\mathbb{F}_{q}^{n}$. Clearly $a=3$ as each right triangle contains 3 points. Each right triangle can be chosen as follows: we first choose two distinct vectors $x, y \in \mathbb{F}_{q}^{n}$, in $\Theta\left(q^{2 n}\right)$ different ways, then we choose a vector $z \in \mathbb{F}_{q}^{n}$ such that

$$
(z-x) \cdot(y-x)=0 \quad \text { or } \quad(z-y) \cdot(x-y)=0 .
$$

Since $\left\{\xi \in \mathbb{F}_{q}^{n}: \xi \cdot(x-y)=0\right\}$ is a $(n-1)$-dimensional subspace, there are $\Theta\left(q^{n-1}\right)$ choices of $z$ such that (17) holds. Thus $|\mathcal{A}|=O\left(q^{3 n-1}\right)$. For a lower bound, observe that each right triangle in $\mathbb{F}_{q}^{n}$ was chosen at most $O(1)$ times using this process. Furthermore, the above process may also produce triples $(x, y, z)$ with $z=x$ or $z=y$, and these are not right triangles. Therefore

$$
\Theta\left(q^{3 n-1}\right) \leq O(1)|\mathcal{A}|+O\left(q^{2 n}\right) .
$$

Since $n \geq 2$ it follows that $|\mathcal{A}|=\Theta\left(q^{3 n-1}\right)$, so we take $b=3$ and $c=1$ as in Table 1 . Furthermore the above arguments also implies that the condition (3) holds for right triangles.

\subsection{Affine planes}

Let $G(n, m)$ be the collection of all $m$-dimensional linear subspaces of $\mathbb{F}_{q}^{n}$, and $\mathcal{A}=$ $A(n, m)$ be the family of all $m$-dimensional planes. It is not hard to obtain (see [8, Theorem 6.3] or the proof of Lemma 4.2(i), below),

$$
|G(n, m)|=\frac{\left(q^{n}-1\right)\left(q^{n}-q\right) \ldots\left(q^{n}-q^{m-1}\right)}{\left(q^{m}-1\right)\left(q^{m}-q\right) \ldots\left(q^{m}-q^{m-1}\right)},
$$

and hence

$$
|G(n, m)|=\Theta_{q, m}\left(q^{m n}\right) .
$$

Since every element of $A(n, m)$ is obtained by translating some $m$-dimensional plane, and $q^{n-m}$ elements of $A(n, m)$ are obtained by translating a given $m$-dimensional plane, we obtain

$$
|A(n, m)|=|G(n, m)| q^{n-m}=\Theta_{q, m}\left(q^{(m+1) n}\right) .
$$


By definition of $t(n, q)=q^{-\frac{(m+1) n}{q^{m}}}$, if $\delta=o(t(n, q))$ then

$$
\mathbb{E}(X)=|A(n, m)| \delta^{q^{m}}=o(1) .
$$

This establishes the negative side of the threshold result.

Note that for any $T, T^{\prime} \in A(n, m)$, the intersection $T \cap T^{\prime}$ is ether empty or a $k$-dimensional plane for some $k=0, \ldots, m$. Recall the definition of $I_{q^{k}}$ from (6).

Lemma 4.2. Using above notations, we obtain the following estimates.

(i) Let $V \in A(n, k)$. Then for $m \geq k$,

$$
|\{W \in A(n, m): V \subset W\}|=|G(n-k, m-k)|=\Theta\left(q^{(m-k) n}\right) .
$$

(ii) For $k=0, \ldots, m$ we have

$$
\left|I_{q^{k}}\right| \leq|A(n, k)||G(n-k, m-k)|^{2}=|A(n, m)|^{2} O\left(q^{-(k+1) n}\right) .
$$

It follows that

$$
\left|I_{\geq 1}\right|=|A(n, m)|^{2} O\left(q^{-n}\right)
$$

and hence

$$
\left|I_{0}\right|=|A(n, m)|^{2}\left(1+O\left(q^{-n}\right)\right) .
$$

(iii) Recall that $\mathbb{E}(X)=|A(n, m)| \delta^{q^{m}}=: \lambda_{\mathcal{A}}$. If $\lambda_{\mathcal{A}} \rightarrow \infty$ or $\lambda_{\mathcal{A}} \rightarrow \lambda$ for some positive $\lambda$, then for any $k=0, \ldots, m-1$,

$$
q^{-n(k+1)} \delta^{-q^{k}}=o(1)
$$

Proof. For (i), we assume that $m>k$ and $V \in G(n, k)$. Observe that to obtain a $m$ dimensional subspace which contains $V$, it is sufficient to choose a set $S=\left\{u_{1}, \ldots, u_{m-k}\right\}$ of $\mathbb{F}_{q}^{n}$ such that $S \cup V$ spans an $m$-dimensional subspace. There are $q^{n}-q^{k}$ choices for $u_{1}$ (all except the vectors from the subspace $V$ ). To choose $u_{2}$, we have $q^{n}-q^{k+1}$ choices to ensure that $u_{2}$ is independent of $\left\{u_{1}\right\} \cup V$, and so on. In the end there are

$$
\left(q^{n}-q^{k}\right)\left(q^{n}-q^{k+1}\right) \ldots\left(q^{n}-q^{m-1}\right)
$$

ways to choose $S$. Note that for any $m$-dimensional subspace which contains $V$, there are

$$
\left(q^{m}-q^{k}\right)\left(q^{m}-q^{k+1}\right) \ldots\left(q^{m}-q^{m-1}\right)
$$

choices of $S$ which generate (or span) the same subspace. It follows that the number of $m$-dimensional subspaces which contain $V$ is

$$
\frac{\left(q^{n}-q^{k}\right)\left(q^{n}-q^{k+1}\right) \ldots\left(q^{n}-q^{m-1}\right)}{\left(q^{m}-q^{k}\right)\left(q^{m}-q^{k+1}\right) \ldots\left(q^{m}-q^{m-1}\right)}=|G(n-k, m-k)|
$$

and applying (18) completing the proof of (i). 
To prove (ii), note that $T$ intersects $T^{\prime}$ at some $k$-dimensional plane for any $\left(T, T^{\prime}\right) \in$ $I_{q^{k}}$. For every $k$-plane there are $|G(n-k, m-k)| m$-planes which contain it, and hence by (i) above we have

$$
\left|I_{q^{k}}\right| \leq|A(n, k)||G(n-k, m-k)|^{2}=|A(n, m)|^{2} O\left(q^{-n(k+1)}\right) .
$$

To establish (iii), since $\lambda_{\mathcal{A}}=|A(n, m)| \delta^{q^{m}}$, by (19) we have

$$
\delta^{q^{m}}=\lambda_{\mathcal{A}} q^{-n(m+1)} \Theta(1) .
$$

Then

$$
\begin{aligned}
q^{n(k+1)} \delta^{q^{k}} & =q^{n(k+1)} \lambda_{\mathcal{A}}^{q^{k-m}} q^{-n(m+1) q^{k-m}} \Theta(1) \\
& =\lambda_{\mathcal{A}}^{q^{k-m}} q^{n\left(k+1-(m+1) q^{k-m}\right)} \Theta(1) .
\end{aligned}
$$

Observe that for any $x \geq 0$ and $q \geq 3$,

$$
\frac{x+1}{q^{x}}>\frac{x+2}{q^{x+1}} .
$$

It follows that $k+1>(m+1) q^{k-m}$ for any $k=0,1, \ldots, m-1$. Define

$$
\alpha=\alpha(q, m)=\min \left\{k+1-(m+1) q^{k-m}: k \in \mathbb{N}, k=0, \ldots, m-1\right\} .
$$

Thus $\alpha>0$. It follows that for any $k=0, \ldots, m-1$,

$$
q^{n(k+1)} \delta^{q^{k}}=\lambda_{\mathcal{A}}^{q^{k-m}} q^{n \alpha} \Omega_{q, m}(1),
$$

completing the proof.

We immediately have the following consequence.

Lemma 4.3. Suppose that $\mathbb{E}(X) \rightarrow \infty$ or $\mathbb{E}(X) \rightarrow \lambda$ for some fixed $\lambda>0$. Then $\mathbb{E}(Y)=o\left(\mathbb{E}(X)^{2}\right)$, so (C2) holds.

Proof. Observe that, using Lemma 4.2(ii),

$$
\mathbb{E}(Y)=\sum_{k=1}^{m-1}\left|I_{q^{k}}\right| \delta^{2 q^{m}-q^{k}}=\mathbb{E}(X)^{2} \sum_{k=1}^{m-1} O\left(q^{-n(k+1)} \delta^{-q^{k}}\right) .
$$

Lemma 4.2(iii) implies that

$$
\sum_{k=1}^{m-1} O\left(q^{-n(k+1)} \delta^{-q^{k}}\right)=o(1)
$$

as required. 
Recall that $t(n, q)=q^{-\frac{(m+1) n}{q^{m}}}$. If $\delta=\omega(t(n, q))$ then $\mathbb{E}(X)=|A(n, m)| \delta^{q^{m}} \rightarrow \infty$. Lemma 4.2(ii) and Lemma 4.3 implies that Lemma 2.1 holds for $m$-dimensional planes. This establishes the coarse threshold result of Theorem 1.3 for $m$-dimensional planes.

For later use, we state the following easy fact as a lemma. It follows directly from Lemma 4.2(i).

Lemma 4.4. Given $F=\left\{x_{1}, \ldots, x_{t}\right\} \subseteq \mathbb{F}_{q}^{n}$, let $d(F)$ be the smallest integer $k$ such that $F \subseteq V$ for some $V \in A(n, k)$. Then for $m \geq d(F)$,

$$
|\{T \in A(n, m): F \subseteq T\}|=|G(n-d(F), m-d(F))|=\Theta_{q, m}\left(q^{n(m-d(F))}\right) .
$$

Now we show that if $\mathbb{E}(X) \rightarrow \lambda$ for some fixed positive $\lambda$ then for any $r \in \mathbb{N}$, $\mathbb{E}\left(X^{r}\right)=O_{r}(1)$. Applying the same argument as in the proof of Theorem 1.1, it is sufficient to prove the following result.

Lemma 4.5. Assume that $\mathbb{E}(X) \rightarrow \lambda$ for some fixed positive $\lambda$. Let $T_{1}, \ldots, T_{r} \in$ $A(n, m)$. Then

$$
\sum_{T \in A(n, m)} \mathbb{P}\left(T \subseteq E \mid \cup_{i=1}^{r} T_{i} \subseteq E\right)=O_{q, m, \lambda, r}(1)
$$

Proof. Let $B=\cup_{i=1}^{r} T_{i}$. For $k=0,1, \ldots, q^{m}$, define

$$
J_{k}=\{T \in A(n, m):|T \cap B|=k\} .
$$

We will split the sum up as follows:

$$
\begin{aligned}
& \sum_{T \in A(n, m)} \mathbb{P}\left(T \subseteq E \mid \cup_{i=1}^{r} T_{i} \subseteq E\right) \\
& \quad=\sum_{k=0}^{q^{m}}\left|J_{k}\right| \delta^{a-k} \\
& =\left|J_{0}\right| \delta^{a}+\left|J_{1}\right| \delta^{a-1}+\sum_{j=1}^{m-1} \sum_{q^{j-1}<k \leq q^{j}}\left|J_{k}\right| \delta^{a-q^{j}}+\sum_{q^{m-1}<k \leq q^{m}}\left|J_{k}\right| \delta^{a-k} .
\end{aligned}
$$

Let $a:=q^{m}$ and $\lambda=\mathbb{E}(X)$. First, $\left|J_{0}\right| \leq|A(n, m)|$ so

$$
\left|J_{0}\right| \delta^{a} \leq|A(n, m)| \delta^{a}=\lambda=O(1) .
$$

Next, by (19),

$$
\left|J_{1}\right| \leq|B||G(n, m)|=O\left(q^{-n}\right)|A(m, n)| .
$$

Applying Lemma 4.2(iii) we have

$$
\left|J_{1}\right| \delta^{a-1}=O\left(q^{-n} \delta^{-1} \lambda\right)=o(1) .
$$

Now let $k \in\left\{2,3, \ldots, q^{m}\right\}$. Then there exists $j \in\{1, \ldots, m\}$ such that $q^{j-1}<k \leq q^{j}$. Let $F \subseteq B$ with $|F|=k$. The smallest plane which contains $F$ has dimension at least $j$. Hence Lemma 4.4 implies that

$$
|\{T \in A(n, m): F \subseteq T\}|=O\left(q^{n(m-j)}\right) .
$$


Since $|B| \leq r q^{m}=O(1)$, there are $\left(\begin{array}{c}|B| \\ k\end{array}\right)=O(1)$ different choices for a subset $F$ consisting of $k$ points of $B$. Therefore, by (19),

$$
\left|J_{k}\right|=O\left(q^{n(m-j)}\right)=O\left(q^{-n(j+1)}\right)|A(n, m)|
$$

and since $q, m$ are fixed, we obtain

$$
\sum_{q^{j-1}<k \leq q^{j}}\left|J_{k}\right|=O\left(q^{-n(j+1)}\right)|A(n, m)| .
$$

If $j \leq m-1$ then applying Lemma 4.2(iii) gives

$$
\begin{aligned}
\sum_{j=1}^{m-1} \sum_{q^{j-1}<k \leq q^{j}}\left|J_{k}\right| \delta^{a-q^{j}} & =\sum_{j=1}^{m-1} O\left(q^{-n(j+1)} \delta^{-q^{j}}\right)|A(n, m)| \delta^{a} \\
& =o(1) .
\end{aligned}
$$

Finally, when $j=m$ we have, by (19) and (24),

$$
\sum_{q^{m-1}<k \leq q^{m}}\left|J_{k}\right| \delta^{a-k} \leq \sum_{q^{m-1}<k \leq q^{m}}\left|J_{k}\right|=O(1)
$$

The result follows by substituting (22), (23), (25) and (26) into (21).

Applying Lemma 4.5 and arguing in the proof of Theorem 1.1, we obtain that condition (C3) from Lemma 2.3 holds for $m$-dimensional planes. Together with Lemma 4.2(ii) and Lemma 4.3, we obtain Lemma $2.3(\mathrm{C} 1)$ and $(\mathrm{C} 2)$, completing the proof of Theorem 1.3 for $m$-dimensional planes.

Extensions and limitations: It is possible that our analysis of $m$-dimensional subspaces in Theorem 1.3 can be extended to allow $m$ or $q$ to grow slowly with $n$. Bounding $|\mathcal{A}|$ more carefully in (19) suggests that the threshold function should be adjusted to $t(n, q)=q^{-\frac{(m+1)(n-m)}{q^{m}}}$ in that case. This function is very close to 1 unless $n$ grows faster than $\frac{q^{m}}{(m+1) \log q}$.

\section{Further results and future directions}

We now give some applications to extremal problems, discuss an Erdős-Rényi variant of the random model and mention some open problems.

\subsection{Applications to extremal problems}

Borrowing notation from extremal graph theory, let $\operatorname{ex}\left(\mathbb{F}_{q}^{n}, \mathcal{A}\right)$ denote the maximal cardinality of subsets of $\mathbb{F}_{q}^{n}$ which are $\mathcal{A}$-free. That is,

$$
\operatorname{ex}\left(\mathbb{F}_{q}^{n}, \mathcal{A}\right)=\max \left\{|B|: B \subseteq \mathbb{F}_{q}^{n}, B \text { does not contain any element of } \mathcal{A}\right\} .
$$


For the random variable $X$, Markov's inequality implies that

$$
\mathbb{P}(X \geq 1) \leq \mathbb{E}(X)=|\mathcal{A}| \delta^{a} .
$$

Chebyshev's inequality implies that for our random subset $E$,

$$
\mathbb{P}\left(|| E\left|-q^{n} \delta\right| \geq \frac{1}{2} q^{n} \delta\right) \leq \frac{4 q^{n} \delta(1-\delta)}{\left(q^{n} \delta\right)^{2}}=\frac{4(1-\delta)}{q^{n} \delta} .
$$

That is, $|E|$ is concentrated around $q^{n} \delta$ when $q^{n} \delta$ is large.

Applying the estimates (27) and (28) leads to the following lower bound on $\operatorname{ex}\left(\mathbb{F}_{q}^{n}, \mathcal{A}_{a}\right)$.

Lemma 5.1. Suppose that $|\mathcal{A}| \delta^{a}=\frac{1}{2}$ and $q^{n} \delta \geq 100$. Then there exists a subset $S \subseteq \mathbb{F}_{q}^{n}$ which is $\mathcal{A}$-free and satisfies $|S|=\Theta\left(q^{n} \delta\right)$. This implies that

$$
\operatorname{ex}\left(\mathbb{F}_{q}^{n}, \mathcal{A}\right)=\Omega\left(q^{n} \delta\right)=\Omega\left(q^{n}|\mathcal{A}|^{-\frac{1}{a}}\right)
$$

This leads to the following lower bounds for the extremal problem for the patterns defined in Definition 1.2.

Corollary 5.2. As usual, let $q \geq 2$ be a prime power and let $n$ be a positive integer. Let $\mathcal{A}_{1}, \mathcal{A}_{2}$ and $\mathcal{A}_{3}$ be the collection of all non-trivial $k$-APs, sums and non-trivial parallelograms in $\mathbb{F}_{q}^{n}$, respectively. Similarly, let $\mathcal{A}_{4}$ denote the collection of all nontrivial right triangles in $\mathbb{F}_{q}^{n}$, where $n \geq 2$, and let $\mathcal{A}_{5}$ denote the collection of all $m$ dimensional planes in $\mathbb{F}_{q}^{n}$, where $m, q$ are fixed and $q \geq 3$. Then

$$
\begin{aligned}
\operatorname{ex}\left(\mathbb{F}_{q}^{n}, \mathcal{A}_{1}\right)=\Omega\left(q^{n\left(1-\frac{2}{k}\right)}\right), & \operatorname{ex}\left(\mathbb{F}_{q}^{n}, \mathcal{A}_{2}\right)=\Omega\left(q^{\frac{n}{3}}\right), \quad \operatorname{ex}\left(\mathbb{F}_{q}^{n}, \mathcal{A}_{3}\right)=\Omega\left(q^{\frac{n}{4}}\right), \\
\operatorname{ex}\left(\mathbb{F}_{q}^{n}, \mathcal{A}_{4}\right)=\Omega\left(q^{\frac{1}{3}}\right), & \operatorname{ex}\left(\mathbb{F}_{q}^{n}, \mathcal{A}_{5}\right)=\Omega\left(q^{n\left(1-\frac{m+1}{q^{m}}\right)}\right) .
\end{aligned}
$$

Note that the extremal problem for 3-APs in $\mathbb{F}_{3}^{n}$ is called the cap set problem, and a much stronger lower bound is known. For the cap set problem, the best known bounds are

$$
2.2^{n} \leq \operatorname{ex}\left(\mathbb{F}_{3}^{n}, 3-\mathrm{AP}\right) \leq 2.756^{n} .
$$

See [16] for the lower bound and [14] for the upper bound (and further background). To our knowledge the other lower bounds appear to be new.

\subsection{Erdős-Rényi model for finite vector spaces}

In this subsection, we consider another random model in $\mathbb{F}_{q}^{n}$ which is an analogue of Erdös-Rényi random graphs. Let $M=M_{n, q} \leq q^{n}$ be a positive integer. Choose $E=E^{\omega}$ uniformly at random from the set of all subsets of $\mathbb{F}_{q}^{n}$ of cardinality $M$. Denote this probability space by $\Omega\left(\mathbb{F}_{q}^{n}, M\right)$.

Note that for a subset $F \subseteq \mathbb{F}_{q}^{n}$ with $|F| \leq M$, we have

$$
\mathbb{P}(F \subseteq E)=\frac{M(M-1) \ldots(M-|F|+1)}{q^{n}\left(q^{n}-1\right) \ldots\left(q^{n}-|F|+1\right)} .
$$


It follows that if $|F|=O(1)$ and $M_{n, q} \rightarrow \infty$ then the identity (29) becomes

$$
\mathbb{P}(F \subseteq E) \sim\left(\frac{M_{n, q}}{q^{n}}\right)^{|F|} .
$$

For the model $\Omega\left(\mathbb{F}_{q}^{n}, M\right)$, we can obtain similar results to Theorems 1.3 by taking the ratio $M_{n, q} / q^{n}$ instead of $\delta$ in our former proofs. We omit these arguments.

\subsection{Future directions}

Our results leave many open questions in the analysis of substructures in random subsets of $\mathbb{F}_{q}^{n}$. Some possible directions for future study are listed below.

- To what extent can condition (3) in Theorem 1.1 be relaxed or removed? Recall that in random graphs, the threshold for existence of a given subgraph $G$ is controlled by the parameter $m(G)$, which is the density of the densest subgraph of $G$. To remove condition (3) it may be necessary to define an analogous parameter for substructures in $\mathbb{F}_{q}^{n}$.

- We have only analysed the limiting distribution of the random variable $X$, defined in (1), in the case that $\mathbb{E} X$ tends to a constant (that is, within the threshold scale). In this situation we proved that the limiting distribution is Poisson (in some cases we could prove this only when $q \rightarrow \infty$ ). A natural question is to ask for conditions under which the limiting distribution of $X$ is normal (see [32] for the random graph setting). This would involve application of the method of moments, or Stein's method.

- A fruitful line of research in recent years (for example [11, 27, 31, 35]), seeks what Conlon and Gowers [11] call "sparse random" analogues of extremal and Ramseytheoretic problems. In our setting, let $\mathcal{A}$ be a given pattern and recall that $E$ is a random subset of $\mathbb{F}_{q}^{n}$ chosen according to $\Omega\left(\mathbb{F}_{q}^{n}, \delta\right)$. One open problem is to find a threshold function for the property that, in every $r$-colouring of $E$, there is a monochromatic element of $\mathcal{A}$. Another aim is to establish, for a given pattern $\mathcal{A}$, a threshold function for the probability that every subset $F \subseteq E$ with $|F| \geq \alpha|E|$ must contain an element of $\mathcal{A}$.

\section{Acknowledgements}

The authors would like to thank the referees for their extremely helpful comments which have greatly improved this paper.

\section{References}

[1] N. Alon and J. Spencer, The Probabilistic Method, Wiley Interscience, New York, 2000 . 
[2] L. Babai. Fourier transforms and equations over finite Abelian groups: An introduction to the method of trigonometric sums. Lecture notes, available from http://people.cs.uchicago.edu/ laci/reu02/fourier.pdf

[3] P. Billingsley, Probability and Measure (3rd ed.), Wiley, New York, 1995.

[4] B. Bollobás, Threshold functions for small subgraphs, Tham. Proc. Camb. Philos. Soc. 90 (1981), 187-206.

[5] B. Bollobás, Random Graphs (2nd ed.), Cambridge University Press, 2001.

[6] B. Bollobás and A. Thomason, Threshold functions, Combinatorica 7(1) (1987), $35-38$.

[7] J. Bourgain, N. Katz and T. Tao, A sum-product estimate in finite fields and their applications, Geom. Func. Anal. 14 (2004), 27-57.

[8] P. Cameron, The Art of Counting. Lecture notes, available from http://cameroncounts.files. wordpress.com/2016/04/acnotes1.pdf

[9] C. Chen, Salem sets in vector spaces over finite fields, Ark. Mat. 56 (2018), 45-52.

[10] C. Chen, Projections in vector spaces over finite fields, Ann. Acad. Sci. Fenn. Math. 43, (2018), 171-185.

[11] D. Conlon and W. T. Gowers, Combinatorial theorems in sparse random sets, Ann. of Math. (2) 184 (2016), 367-454.

[12] Z. Dvir, On the size of Kakeya sets in finite fields, J. Amer. Math. Soc 22 (2009), 1093-1097.

[13] J. Ellenberg and D. Erman. Furstenberg sets and Furstenberg schemes over finite fields. Algebra and Number Theory, 10 (2016), 1415-1436.

[14] J. Ellenberg and D. Gijswijt, On large subsets of $\mathbb{F}_{q}^{n}$ with no three-term arithmetic progression, Ann. of Math. (2) 185 (2017), 339-343.

[15] P. Erdös, A. Rényi, On the evolution of random graphs, Publications of the Mathematical Institute of the Hungarian Academy of Sciences, 5 (1960), 17-61.

[16] Y. Edel, Extensions of generalized product caps, Des. Codes Cryptogr. 31(1) (2004), 5-14.

[17] K. Falconer, Fractal Geometry: Mathematical Foundation and Applications, Wiley, New York, 1990.

[18] A. Frieze and M. Karoński, Introduction to Random Graphs, Cambridge University Press, Cambridge, 2015.

[19] E. Friedgut, V. Rödl and M. Schacht, Ramsey properties of random discrete structures, Random Structures Algorithms 37 (2010), 407-436. 
[20] H. Furstenberg and Y. Katznelson, An ergodic Szemerédi theorem for IP-systems and combinatorial theory, J. Analyse Math. 45 (1985), 117-168.

[21] R. Graham, V. Rödl and A. Ruciński, On Schur properties of random subsets of integers, J. Number Theory 61 (1996), 388-408.

[22] B. Green, Restriction and Kakeya phenomena, Lecture notes (2003).

[23] B. Green, Finite field models in additive combinatorics, in Surveys in Combinatorics 2005, London Mathematical Society Lecture Note Series, Volume 327, pp. 1-27 (Cambridge University Press, 2005).

[24] A. Iosevich and M. Rudnev, Erdős distance problem in vector spaces over finite fields, Trans. Amer. Math. Soc, 359 (2007), 6127-6142.

[25] M. Karoński and A. Ruciński, On the number of strictly balanced subgraphs of a random graph, in Graph Theory, Proceedings Łagów 1981, Lecture Notes in Mathematics vol. 1018, Springer, Berlin, 1983, pp. 79-83.

[26] J.-H. Kim, B. Sudakov and V. Vu, Small subgraphs of random regular graphs, Discrete Matheamtics 307 (2007), 1961-1967.

[27] Y. Kohayakawa, T. Łuczak and V. Rödl, Arithmetic progressions of length three in subsets of a random set, Acta Arith. 75 (1996), 133-163.

[28] Y. Kohayakawa, S. J. Lee, V. Rödl and W. Samotij, The number of Sidon sets and the maximum size of Sidon sets contained in a sparse random set of integers, Random Structures Algorithms 46 (2015), 1-25.

[29] G. Mockenhaupt and T. Tao, Restriction and Kakeya phenomena for finite fields, Duke Math. J., 121 (2004), 35-74.

[30] V. Rödl and A. Ruciński, Threshold functions for Ramsey properties, J. Amer. Math. Soc. 8 (1993), 917-942.

[31] V. Rödl and A. Ruciński, Rado partition theorem for random subsets of integers, Proc. London Math. Soc. 74 (1997), 481-502.

[32] A. Ruciński, When are small subgraphs of a random graph normally distributed? Probab. Theory Related Fields. 78 (1988), 1-10.

[33] A. Ruciński and A. Vince, Strongly balanced graphs and random graphs, J. Graph Theory 10 (1986), 251-264.

[34] J. Rué, C. Spiegel and A. Zumalacárregui, Threshold functions and Poisson convergence for systems of equations in random sets, Math. Z. 288 (2018), 333-360.

[35] M. Schacht, Extremal results for random discrete structures, Ann. of Math. (2) 184 (2016), 333-365. 
[36] P. Shmerkin and V. Suomala, Patterns in random fractals, to appear in Amer. J. Math.. arXiv: 1703.09553

[37] T. Tao and V. Vu, Additive Combinatorics, Cambridge Univ. Press, 2006.

[38] J. Wolf, Finite field models in arithmetic combinatorics-ten years on, Finite Fields Appl. 32 (2015), 233-274.

[39] T. Wolff, Recent work connected with the Kakeya problem. Prospects in mathematics (Princeton, NJ, 1996), (1999), 129-162. 